\title{
LINEAR PLASMA DEVICE PSI-2 FOR PLASMA-MATERIAL INTERACTION STUDIES
}

\author{
A. Kreter ${ }^{*}$, C. Brandt, A. Huber, S. Kraus, S. Möller, M. Reinhart, B. Schweer, G. Sergienko, \\ B. Unterberg \\ Forschungszentrum Jülich GmbH, Institut für Energie- und Klimaforschung - Plasmaphysik, \\ 52425 Jülich, Germany \\ *Email address: a.kreter@,fz-juelich.de
}

The linear plasma device PSI-2 serves as a pilot experiment for the development of components, operational regimes and control systems for the linear plasma device JULE-PSI, which will be located in the nuclear environment allowing studies of radioactive and toxic samples. PSI-2 is also used for fusion reactor relevant plasma-material interaction studies. This contribution describes the PSI-2 layout and parameters and summarizes the recent scientific and technical progress in the project, including the installation of a target station for the sample manipulation and analyses.

Keywords: plasma facing material; plasma-material interaction; PSI-2; JULE-PSI

\section{INTRODUCTION}

Plasma-material interaction (PMI) processes can lead to reduced availability of a fusion reactor through the shortened lifetime of the wall components and the limitations by safety regulations, including the restricted amount of tritium retention. ${ }^{1}$ Linear plasma devices are excellent test beds for investigating specific questions of PMI. The materials can be tested under well-defined plasma exposure conditions relevant to fusion edge plasma. $^{2}$

The linear plasma device PSI-2 was initially constructed and operated at the Humboldt University in Berlin. ${ }^{3}$ The device was transferred to Forschungszentrum Jülich and resumed the 
plasma operation in 2011. PSI-2 is a pilot experiment of the JULE-PSI project, which foresees a linear plasma device installed in the nuclear environment for the operation with neutron irradiated and toxic materials. ${ }^{4}$ The main aims of PSI-2 in Jülich are: (i) development and testing of components and technological solutions for JULE-PSI such as plasma production, diagnostics and sample manipulation, (ii) training of the personnel operating linear plasma device and (iii) contributing to the PMI research for ITER and future fusion reactors.

This contribution describes the current state of the PSI-2 device. The general layout of PSI-2 including main components and diagnostics is given in section II. The main focus is laid on the recently installed target station, a sample manipulator with a target exchange and analysis chamber and a linear drive (section III). Examples of the ongoing PMI research are presented in section IV.

\section{LAYOUT, DIAGNOSTICS AND PLASMA PARAMETERS OF PSI-2}

The plasma chamber of PSI-2 is surrounded by six magnetic field coils (Fig. 1) and includes the plasma source, the buffer vacuum region and the target exposure chamber. Plasma is created by an arc discharge between a heated $\mathrm{LaB}_{6}$ cathode emitter and a hollow molybdenum anode. A working gas flow of $\sim 10-100 \mathrm{sccm}$ creates a neutral gas pressure of a few Pa necessary for the optimal operation of the plasma source. The standard working gas is deuterium, however, the source can be operated with other, i.e. noble, gases and a mixture of them. The outer edge of the cylindrical cathode is connected to the inner surface of the anode along the magnetic field lines created by the first two coils. In this area the arc discharge "burns" parallel to the magnetic field lines, unlike in the central part, where the discharge occurs across the B field. Therefore, most of the arc power in this configuration is released in the outer radial region resulting in a hollow plasma profile (Fig. 2). Research and development studies are ongoing to upgrade the source and 
ensure a flat plasma profile over several $\mathrm{cm}$ with the maximum on the radial axis. A flat plasma profile is more favorable for the PMI studies since it ensures homogeneous conditions across the exposed material samples and significantly simplifies the measurements by spectroscopy.

The third coil roughly doubles the magnetic field to $280 \mathrm{mT}$. The narrowed plasma column goes here through a gas diaphragm, while most of the injected gas is pumped down by a turbomolecular pump attached to the vacuum port between coils 2 and 3 . This differential pumping system decouples the plasma production from the target exposure region, where different gas mixtures and pressures (down to $\sim 10^{-2} \mathrm{~Pa}$ ) can be realized by separate gas inlet and pump systems. In the exposure chamber a plasma column with a diameter of $\approx 6 \mathrm{~cm}$ is sustained by a field of $100 \mathrm{mT}$ produced by coils 4 and 5 connected in series. Coil 6 increases the field back to $280 \mathrm{mT}$ to reduce the plasma diameter at the retractable dump placed further downstream. The exposure chamber consists of three segments between coils $3 / 4,4 / 5$ and $5 / 6$ equipped with vacuum ports for plasma and PMI diagnostics. Most of the sample exposure experiments are carried out in the middle segment between coils $4 / 5$, which also provides two diagonal ports for the front observation of the target. Samples are introduced into the exposure chamber by the side-fed sample manipulator or the target station. Radially the samples are usually placed at the maximum of the flux, $2.5-3 \mathrm{~cm}$ off the plasma axis. The total length of the plasma chamber is $\approx 3$ $\mathrm{m}$. The target station roughly doubles the length to $\approx 6 \mathrm{~m}$.

PSI-2 operates at a magnetic field of $100 \mathrm{mT}$ in the target exposure region. At this field, the heavy impurity ions like tungsten are not magnetized. This limits the applicability of the device concerning the studies of particle transport relevant issues of plasma-surface interaction. However, the questions of plasma-material interaction crucial for the availability of the future fusion reactor, like erosion and fuel retention of the first wall materials, can be treated at these 
conditions.

Radial profiles of electron density and temperature are measured by a movable Langmuir double probe installed at the top of the target exposure chamber. The probe moves radially with a velocity of $15 \mathrm{~mm} / \mathrm{s}$, while the $\mathrm{I}-\mathrm{V}$ characteristic is scanned with a frequency of $100 \mathrm{~Hz}$. The radial resolution of the measurements is given by the size of the probe tips of $1.5 \mathrm{~mm}$. In addition, a Thomson scattering system for $n_{e}, T_{e}$ measurements is under construction, which has an advantage of no moving parts inside the vacuum chamber. Optical emission spectroscopy is used for the characterization of the main plasma, ${ }^{5,6}$ impurity ion concentration ${ }^{7-9}$ and in-situ characterization of the PMI processes such as erosion from the target. ${ }^{10}$ An in-situ quartz microbalance system is used to measure the amount of the re-deposition of material sputtered from the sample surface. Sample temperature is controlled by thermocouples placed at the rear side of the sample and by infrared techniques of the front surface. After exposure, the samples are analysed by a number of surface analysis techniques. Some of the surface analysis can be done in-vacuo in the target exchange and analysis chamber of the target station (Sec. III).

The main operational parameters of PSI-2 are summarized in Table I. Fig. 2 shows the radial profiles of electron density, temperature and ion flux measured by the Langmuir probe for a variation of the arc power. A constant deuterium flow of $70 \mathrm{sccm}$ resulted in a neutral gas pressure in the source of $1.5 \mathrm{~Pa}$. While the temperature is not significantly affected, the density and, resultantly, the flux scale approximately linearly with the arc power. The scan of the arc power in Fig. 2 was limited to $26 \mathrm{~kW}$ because the operation at a higher level of power significantly reduces the lifetime of the source. In the past, the source was operated at a power of about $100 \mathrm{~kW}$ to obtain ion fluxes of $\sim 10^{23} \mathrm{~m}^{-2} \mathrm{~s}^{-1}$. The electron temperature can effectively be controlled by the amount of gas injected in the source. E.g. at a constant arc power of $11 \mathrm{~kW}$ the 
maximum temperature can be varied between $8 \mathrm{eV}$ for $200 \mathrm{sccm} \mathrm{D}_{2}$, corresponding to a pressure in the source of $5.8 \mathrm{~Pa}$, and $25 \mathrm{eV}$ for $25 \mathrm{sccm} \mathrm{D}_{2}(0.4 \mathrm{~Pa})$. With this two "knobs", the arc power and the gas inlet, the plasma parameters can be varied in a broad range. The magnetic field configuration in the source also affects the plasma parameters.

\section{TARGET STATION}

A major task of PSI-2 in Jülich in view of the JULE-PSI project is the development of a sample transport system that allows the positioning of the target at three segments inside the exposure chamber without breaking the main vacuum and interrupting the discharge. With this set-up various diagnostics occupying different ports in the three sections can be used.

This requested operational scenario requires a movement of the target along the $\mathrm{z}$ (plasma) axis. Based on this, further tasks were implemented into the project. The target can be exchanged in a separate target exchange and analysis chamber (TEAC) without breaking the main vessel vacuum. The TEAC also serves for sample analysis where the axes of all ports equipped with surface analysis diagnostics are focussed to the centre of the spherically shaped chamber. The optimal conditions for the application of many diagnostics require the orientation of the target normal towards the selected port axis. Therefore the carrier head is rotatable around and tiltable perpendicular the $\mathrm{z}$ axis. It has an interface for the insertion of an exchangeable target holder. Further features are implemented into the carrier head. The temperature can be measured with $\mathrm{Ni}-\mathrm{NiCr}$ (Type K) thermocouples and insulated wires allow the application of electrical probes and resistive electrical heating in the exchangeable target holder. A tube in the target holder gives the possibility of the gas injection through the target. Fig. 3 presents the design with major components of the TEAC. The notation of vacuum ports corresponds to their orientation with respect to the $\mathrm{x}, \mathrm{y}$ and $\mathrm{z}$ axes with $\mathrm{z}$ being the plasma axis and $\mathrm{x}$ (horizontal) and $\mathrm{y}$ (vertical) are 
the axes perpendicular to the plasma column, as indicated in Fig. 1. E.g. the $\mathrm{Z}$ ports are oriented parallel to the $\mathrm{z}$ axis, the $\mathrm{XZ}$ ports are in the $\mathrm{xz}$ plane with $45^{\circ}$ to both $\mathrm{x}$ and $\mathrm{z}$ axes and the $\mathrm{XYZ}$ ports are under $45^{\circ}$ to all three axes.

\section{III.A. Vacuum System of Target Station}

The central component of the TEAC is a spherical vessel with 0.4 m diameter equipped with 13 Conflat (CF) flanges of different sizes. Additionally three CF63 flanges with removable support pistons are distributed equidistantly around the Z-2 flange that is connected to the linear motion system and indicated in fig. 3. The target holder passes the chamber through the ports Z-2 and Z-1 in z direction. A gate valve V2 is mounted at the Z-1 flange followed by an intermediate volume, which contains an aperture with vacuum sealing to the carrier tube, three pistons with spheres in fixed radial positions and a separate port (CF35) for pumping. A second gate valve V1 is the interface to the plasma chamber, which is mechanically decoupled by an edge welded bellow. The advantage of this arrangement is the separation of the two vacuum chambers at any operational condition, as described in Sec. III.D. A water cooled plasma dump made of copper can be inserted by a piston from the bottom side into the plasma chamber between coil 6 and the interface gate valve V1. The dump must be retracted when the target is inserted for the plasma exposure. Turbo pump systems with 1500 ltr/s and 50 lts/s are connected with the target chamber and the intermediate volume, respectively. Both can be decoupled by gate valves.

\section{III.B. Central Carrier}

A tube of $3 \mathrm{~m}$ length carrying the manipulator head is inserted in an edge welded bellow system of $150 \mathrm{~mm}$ inner diameter. The tube is connected at the rear end with a gimbal system mounted on a sledge of the linear transport system. The second support for the carrier is located in the port Z-2 by three pneumatic driven pistons with ceramic spheres allowing translation in and rotation 
around $\mathrm{z}$ direction. They are retracted about $1 \mathrm{~mm}$ radially when the carrier head on its way towards the plasma chamber passes the fixed piston with spheres in the intermediate chamber and vice versa. The maximum values for linear positioning and rotation are $2.5 \mathrm{~m}$ and $\pm 180^{\circ}$, respectively. The speed of the lateral movement is up to $0.3 \mathrm{~m} / \mathrm{s}$ with an accuracy of $\pm 50 \mu \mathrm{m}$, which is determined by the ball bearing spindle drive. An electrical motor rotates the tube within $10 \mathrm{~s}$ for one full circle with an accuracy of $1^{\circ}$. The carrier is electrically insulated from the ground potential at the tube supports and can be biased up to $300 \mathrm{~V}$.

The manipulator head is mounted on a CF flange, which is fixed with a gasket on the central tube. All feedthroughs between the air atmosphere and ultra-high vacuum are inserted on this flange. It contains a connector for five type $\mathrm{K}$ thermocouples, a connector with three pins for electrical probes and resistive heater, a rotary feedthrough with Viton ring sealing, two tubes with Swagelok connectors for the water cooling circuit, three tubes for the control vacuum at the rotary tube axles and one tube for gas supply at the target (Fig. 4).

\section{III.C. Manipulator Head and Exchangeable Target Holder}

The manipulator head mounted on the carrier tube is basically a water cooled copper block with dovetail guide as socket for the exchangeable target holder. This block can be tilted by $135^{\circ}$ from the position of the surface normal parallel to $\mathrm{z}$ axis. Along with the $\mathrm{z}$ rotation, it makes orientations towards all TEAC ports possible. The tilting axle of the copper block is driven over a bevel gear box by an AC motor at the end of the tube. The rotation speed is $1 \mathrm{rpm}$ with a positioning accuracy of $\pm 2^{\circ}$ caused mainly by the $3 \mathrm{~m}$ long axle between motor and gear box. It is planned to be improved by a Piezo motor at the vacuum feedthrough, which can be operated in the magnetic field. Flexible wires connect the feedthroughs at vacuum side with a 15 pins plug at 
the tiltable $\mathrm{Cu}$ block. To avoid water leakage in the cooling circuit at the rotatable tube axle two quad ring sealings are integrated at each side. The intermediate volumes are pumped separately and controlled by a vacuum gauge. The gas supply tube is closed by a valve outside the vacuum and can be opened by a gate trigger. Fig. 5 gives a view on the target holder head with the $\mathrm{Cu}$ block tilted by $45^{\circ}$.

The principle design of the target station recognizes already the application in the future linear plasma experiment JULE-PSI in the nuclear environment. Samples with different sizes have to be mounted on individually adopted holder with a standardized interface fitting (dovetail guide) into the manipulator head. In a radioactive environment this must be performed remotely. Each individual target holder is equipped with five multi contact stripes, which guarantee a good electrical and thermal conduction to the water cooled $\mathrm{Cu}$ block. A steady-state heat flow of up to $500 \mathrm{~W}$ can be removed through the system. The samples are fixed at the holder by a bolted mask. Several types of sample holders with various degrees of thermal contact to the copper base have been developed for different ranges of the sample temperature. Fig. 6 shows a photograph of a sample holder with a reduced thermal contact designed for operations at temperatures above $600^{\circ} \mathrm{C}$.

\section{III.D. Control and Operation of Target Station}

The target station is controlled by Programmable Logic Control (PLC), which also communicates with the central control of the linear plasma device. The PLC system SIMATIC S7 with PROFIBUS and the WIN CC visualisation is used.

For the target exchange the central carrier is retracted to a predefined position in the TEAC. A manual command opens a valve to vent the vacuum chamber with nitrogen gas. The targets are 
exchanged manually using appropriate tools. The evacuation of the vacuum chamber is automated. When the base pressure is below $10^{-4} \mathrm{~Pa}$, the target exposure mode can be selected.

If the target exposure mode is initiated, the valve V2 (Fig. 3) is opened and the linear motion system positions the manipulator head in the intermediate volume. It stops when it has passed the vacuum sealing. The pistons of the removable carrier support at Z-2 are retracted to avoid stresses to the tube by hyperstatic supports. Immediately after the gate valve V1 is opened and the carrier moves towards the preselected position in the plasma chamber. Simultaneously with the V1 action the retraction of the plasma dump is initiated. The dump must activate an end switch before the target holder approaches its $\mathrm{z}$ position, otherwise the manipulator motion is stopped. During plasma exposure the sample can be moved at any position inside the three segments provided for experiments. The operation of diagnostics can be coupled with the linear motion of the target, e. g. to measure the $\mathrm{z}$ dependence of certain quantities.

If the experiment requires the surface analysis of the sample during a running plasma discharge, the system can be retracted in a reversed sequence. The plasma operation can be continued and the plasma flow is taken by the inserted dump. Eventually, only the bias voltage is switched off. The much lower vacuum pressure in the TEAC is sustained at all times and all diagnostics can be applied immediately when the central carrier has reached the TEAC.

\section{PLASMA-MATERIAL INTERACTION STUDIES ON PSI-2}

Besides its purpose as the pilot experiment of the JULE-PSI project, PSI-2 is actively used for fusion relevant plasma-material interaction studies. Currently the metallic wall materials such as tungsten and its alloys, aluminium as beryllium surrogate and low activation steels are in the focus of investigations. The studies can be done under the variation of i.e. plasma species, 
incident ion flux and fluence, incident ion energy and sample temperature. Pulsed laser irradiation is used for the simulation of transient heat loads in fusion plasma.

In collaboration with the linear plasma device Magnum-PSI ${ }^{11}$ the surface morphology and deuterium retention in tungsten has been investigated under the variation of the incident ion flux, revealing that the relation of retention for low and high fluxes is temperature dependent. ${ }^{12,13}$ The influence of helium and argon on the deuterium retention in tungsten has been investigated at a moderate exposure temperature of $380 \mathrm{~K} .{ }^{9}$ The $\mathrm{D}$ retention drops by a factor of 3-4 if $\mathrm{He}$ is added to plasma. The addition of argon increases the D retention by $\sim 30 \%$.

In collaborative experiments with the linear plasma device PISCES-B ${ }^{14}$ aluminium has exhibited similar phenomena to beryllium with respect to the evolution of the surface morphology and sputtering when exposed to deuterium and mixed deuterium-argon and deuterium-helium plasmas. ${ }^{15}$ However, the thermal desorption characteristics of the aluminium targets has indicated different deuterium retention mechanisms than in beryllium.

The laser technique has been applied to simulate ELM-like thermal shock events combined with the deuterium plasma exposure on tungsten. The $\mathrm{W}$ damage behaviour strongly depends on the loading conditions and the sequence of the particle and heat flux exposures. ${ }^{16}$

The ERO code has been modified for simulating of plasma-material interaction and impurity transport in PSI-2. ${ }^{10}$ Experiments with tungsten targets exposed to argon plasma have been taken as a benchmark for the new version of the code. The optical emission modelled by the code is in a good agreement with the spectroscopy data.

\section{SUMMARY}

The linear plasma device PSI-2 serves in Forschungszentrum Jülich as the pilot experiment of 
the JULE-PSI project, which foresees a linear plasma device in the nuclear environment. A new target station, a linear sample manipulator combined with a target exchange and analysis chamber, was designed, constructed and taken into operation on PSI-2. An intensive experimental programme focusing on metallic plasma-facing materials has been launched to contribute to the fusion relevant PMI research.

\section{REFERENCES}

1. Progress in the ITER Physics Basis, Nucl. Fusion, 47, (2007).

2. A. KRETER, "Reactor-Relevant Plasma-Material Interaction Studies in Linear Plasma Devices," Fusion Sci. Technol., 59, 51 (2011).

3. P. KORNEJEW et al., "Chemical Erosion of CFC at High Ion Flux Densities," Phys. Scr., T91, 29 (2001).

4. B. UNTERBERG et al., "New Linear Plasma Devices in the Trilateral Euregio Cluster for an Integrated Approach to Plasma Surface Interactions in Fusion Reactors," Fusion Eng. Des., 86, 1797 (2011).

5. A. POSPIESZCZYK et al., "Spectroscopic Characterisation of the PSI-2 Plasma in the Ionising and Recombining State," J. Nucl. Mater., 438, S1249 (2013).

6. M. REINHART et al., "Using the Radiation of Hydrogen Atoms and Molecules to Determine Electron Density and Temperature in the Linear Plasma Device PSI-2," Fusion Sci. Technol., 63, 201 (2013).

7. A. KRETER et al., "Mitigation of Carbon Erosion in Beryllium Seeded Deuterium Plasma under Bombardment by Argon and Helium Ions in PISCES-B," J. Nucl. Mater., 417, 651 (2011).

8. D. NISHIJIMA et al., "Spectroscopic Determination of the Singly Ionized Helium Density in Low Electron Temperature Plasmas Mixed with Helium in a Linear Divertor Plasma Simulator," Phys. Plasmas, 14, 103509 (2007).

9. M. REINHART et al., "Influence of Plasma Impurities on the Deuterium Retention in Tungsten Exposed in the Linear Plasma Generator PSI-2," J. Nucl. Mater. (2014), http://dx.doi.org/10.1016/j.jnucmat.2014.11.045.

10. E. MARENKOV et al., "Modeling of Tungsten Transport in PSI-2 with the 3D Monte-Carlo Code ERO," J. Nucl. Mater. (2014), http://dx.doi.org/10.1016/j.jnucmat.2014.10.041. 
11. G. DE TEMMERMAN et al., "High Heat Flux Capabilities of the Magnum-PSI Linear Plasma Device," Fusion Eng. Des., 88, 483 (2013).

12. L. BUZI et al., "Influence of Particle Flux Density and Temperature on Surface Modifications of Tungsten and Deuterium Retention," J. Nucl. Mater., 455, 316 (2014).

13. L. BUZI et al., "Influence of Tungsten Microstructure and Ion Flux on Deuterium Plasma-Induced Surface Modifications and Deuterium Retention," J. Nucl. Mater. (2015), http://dx.doi.org/10.1016/j.jnucmat.2014.12.006.

14. R. P. DOERNER, M. J. BALDWIN and K. SCHMID, "The Influence of a Beryllium Containing Plasma on the Evolution of a Mixed-Material Surface," Phys. Scr., T111, 75 (2004).

15. A. KRETER et al., "Erosion, Formation of Deposited Layers and Fuel Retention for Beryllium under the Influence of Plasma Impurities," Phys. Scr., T159, 014039 (2014).

16. A. HUBER et al., "Investigation of the Impact of Transient Heat Loads Applied by Laser Irradiation on ITER-Grade Tungsten," Phys. Scr., T159, 014005 (2014). 
Table title and figure captions:

TABLE I. Main operational parameters of PSI-2.

Fig. 1. PSI-2 layout including the target station.

Fig. 2. Radial profiles of (a) electron density, (b) electron temperature and (c) ion flux measured by the reciprocating Langmuir probe for a constant deuterium gas flow into the source of $70 \mathrm{sccm}$ resulting in a neutral gas pressure in the source of $1.5 \mathrm{~Pa}$. The arc power was varied as indicated in the legend. The profiles were smoothed using the Savitzky-Golay method, which did not affect the profile shapes. The error bars indicate the statistical deviation of 10 original data points obtained every $10 \mathrm{~ms}$ from the mean value, corresponding to the radial resolution of the probe of $1.5 \mathrm{~mm}$. Note that the maximum is not on the radial axis resulting in a hollow plasma profile.

Fig. 3. Target exchange and analysis chamber (TEAC). Vacuum ports are named according to their orientation as indicated in Fig. 1 and described in the text.

Fig. 4. Interface of the sample manipulator head.

Fig. 5. Manipulator head with $45^{\circ}$ tilted copper block.

Fig. 6. Sample holder designed for the high temperature operation during exposure to deuterium plasma. 
TABLE I. Main operational parameters of PSI-2.

\begin{tabular}{|l|l|}
\hline $\begin{array}{l}\text { Magnetic field in } \\
\text { exposure chamber }\end{array}$ & $0.1 \mathrm{~T}$ \\
\hline $\begin{array}{l}\text { Diameter of } \\
\text { plasma column }\end{array}$ & $\approx 6 \mathrm{~cm}$ \\
\hline Arc current & $\leq 1000 \mathrm{~A}$ \\
\hline Arc voltage & $\leq 150 \mathrm{~V}$ \\
\hline $\begin{array}{l}\text { Electron } \\
\text { temperature }\end{array}$ & $\begin{array}{l}1-25 \mathrm{eV} \text { (in D) } \\
\text { up to } 40 \mathrm{eV} \text { (in He) }\end{array}$ \\
\hline Ion temperature & $0.5-5 \mathrm{eV}$ \\
\hline Electron density & $\sim 10^{17}-10^{19} \mathrm{~m}^{-3}$ \\
\hline Ion flux & $\sim 10^{21}-10^{23} \mathrm{~m}^{-2} \mathrm{~s}^{-1}$ \\
\hline $\begin{array}{l}\text { Incident ion energy } \\
\text { (target bias) }\end{array}$ & $10-300 \mathrm{~V}$ \\
\hline
\end{tabular}




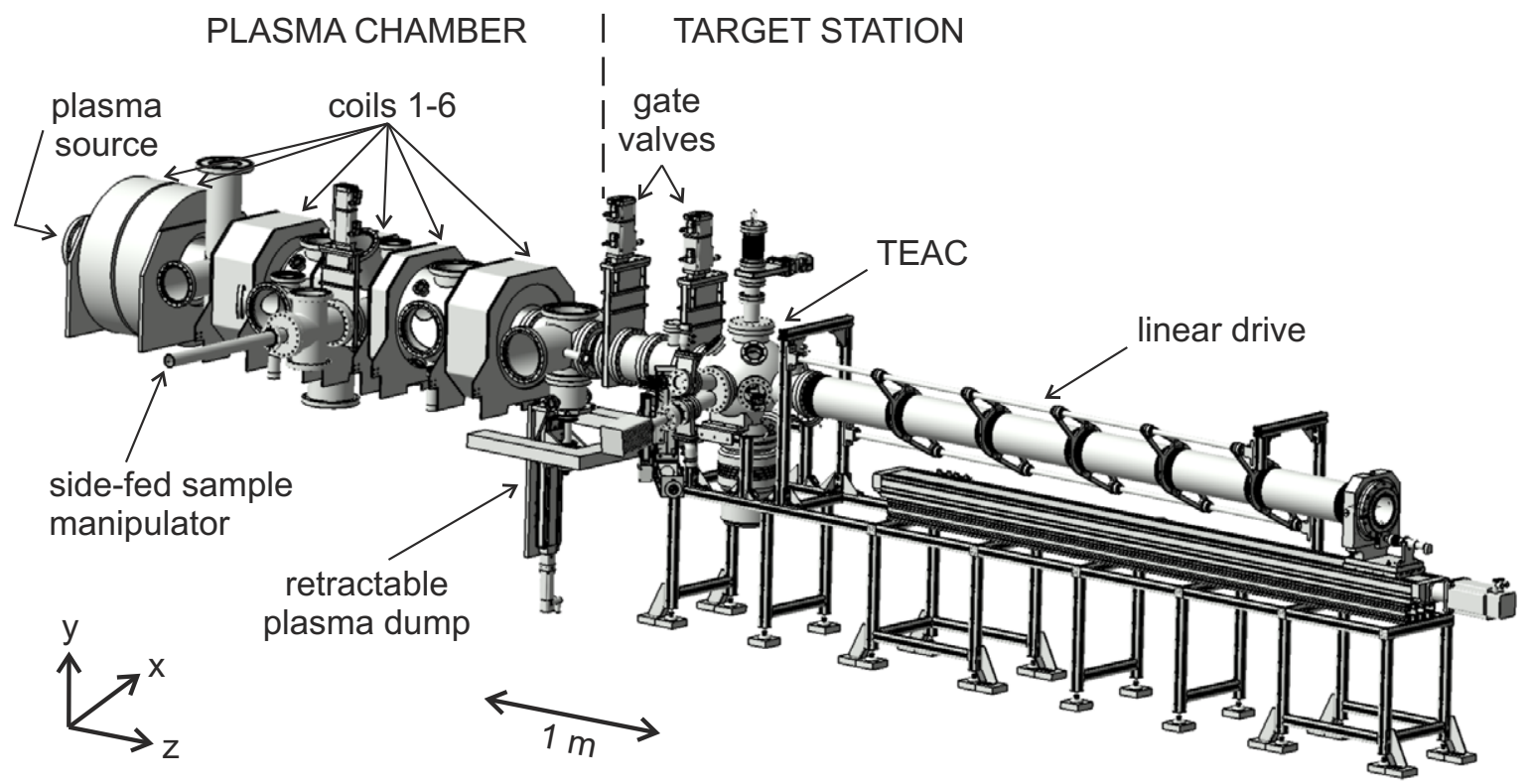

Fig. 1. PSI-2 layout including the target station. 


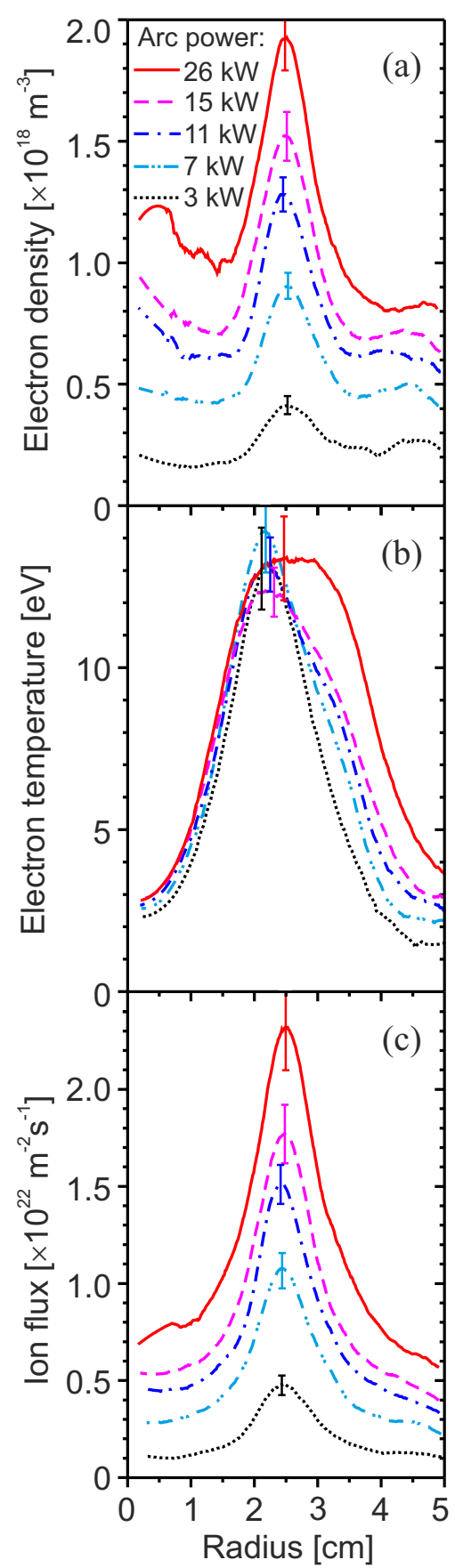

Fig. 2. Radial profiles of (a) electron density, (b) electron temperature and (c) ion flux measured by the reciprocating Langmuir probe for a constant deuterium gas flow into the source of $70 \mathrm{sccm}$ resulting in a neutral gas pressure in the source of $1.5 \mathrm{~Pa}$. The arc power was varied as indicated in the legend. The profiles were smoothed using the Savitzky-Golay method, which did not affect the profile shapes. The error bars indicate the statistical deviation of 10 original data points obtained every $10 \mathrm{~ms}$ from the mean value, corresponding to the radial resolution of the probe of $1.5 \mathrm{~mm}$. Note that the maximum is not on the radial axis resulting in a hollow plasma profile. 


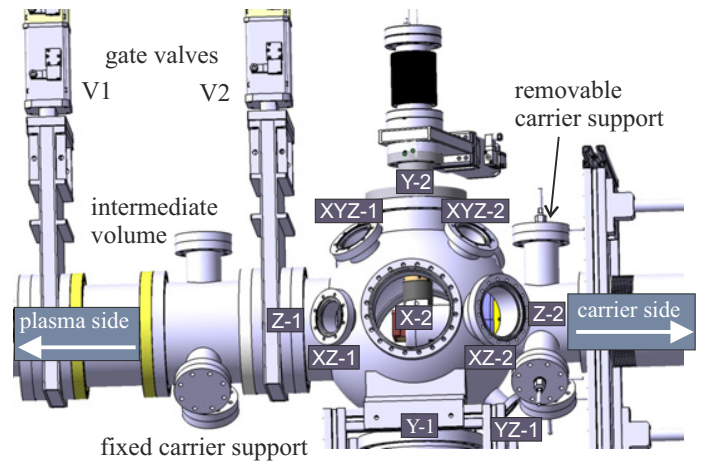

Fig. 3. Target exchange and analysis chamber (TEAC). Vacuum ports are named according to their orientation as indicated in Fig. 1 and described in the text. 


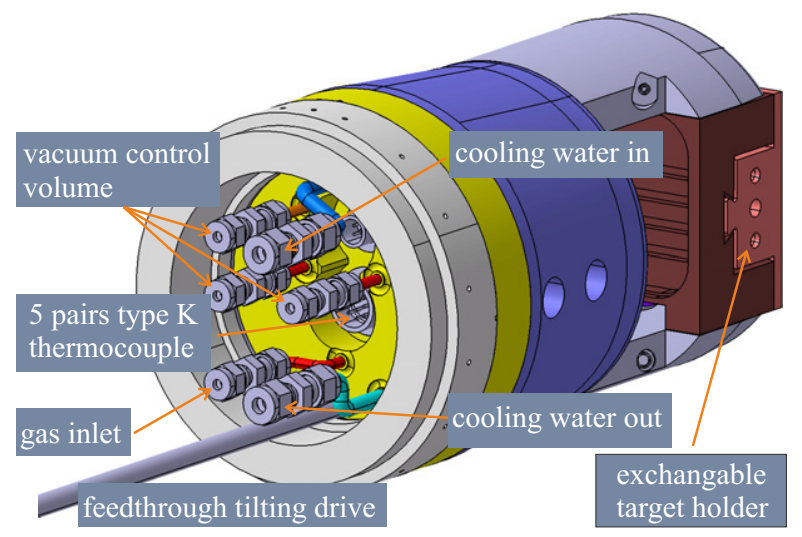

Fig. 4. Interface of the sample manipulator head. 


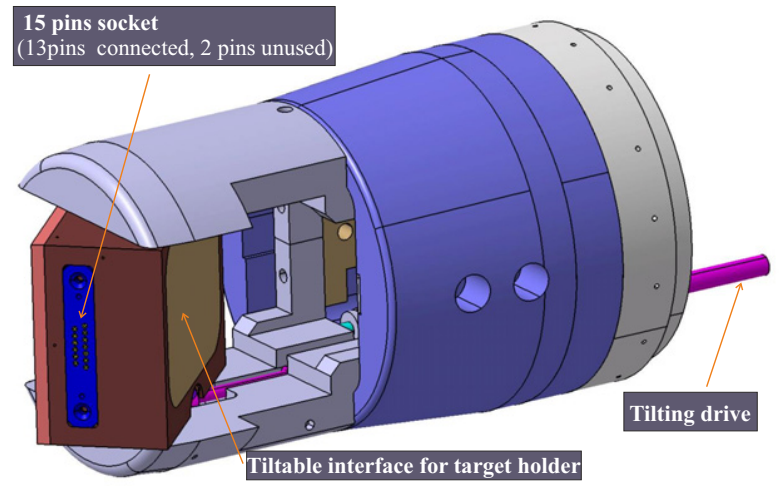

Fig. 5. Manipulator head with $45^{\circ}$ tilted copper block. 


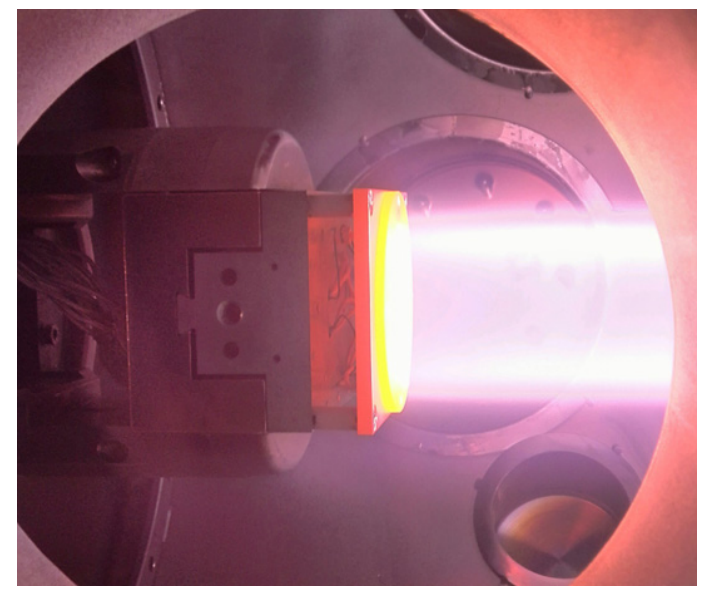

Fig. 6. Sample holder designed for the high temperature operation during exposure to deuterium plasma. 